Referencia para citar este artículo: Ortiz de Zárate, A. \& Guarda, M. N. (2017). Enseñanza contextualizada para miembros de la subcultura del heavy metal a través de técnicas teatrales. Revista Latinoamericana de Ciencias Sociales, Niñez y Juventud, 15 (2), pp. 1035-1050. DOI:10.11600/1692715x.1521611072016

\title{
Enseñanza contextualizada para miembros de la subcultura del heavy metal a través de técnicas teatrales*
}

\author{
AMALIA ORTIZ DE ZiRATE** \\ Profesora Universidad Austral de Chile, Chile. \\ MARÍA NICOL GUARDA *** \\ Profesora Universidad Austral de Chile, Chile.
}

\section{Artículo recibido en julio 11 de 2016; artículo aceptado en septiembre 16 de 2016 (Eds.)}

- Resumen (descriptivo): este artículo da cuenta de la investigación cualitativa que pretende incluir a la subcultura del metal en el aula de inglés como lengua extranjera (ILE) en Chile, utilizando la enseñanza contextualizada y las técnicas teatrales. En primer lugar, se otorgan algunos antecedentes relacionados con el ILE en Chile. En segundo lugar, se desarrolla una revisión bibliográfica, en la que se describe a las subculturas juveniles, los metaleros como subcultura, la motivación para aprender ILE, la enseñanza contextualizada y finalmente las técnicas dramáticas. En tercer lugar se entrega la metodología de investigación utilizada (entrevistas y observación no participante) y algunos de los resultados obtenidos. En último lugar, se presentan conclusiones y una propuesta pedagógica que se sirve de esta investigación para incluir la subcultura juvenil del metal en el aula de ILE.

Palabras clave: subcultura, juventud, enseñanza de una segunda lengua, lengua extranjera, inglés, drama (Tesauro de Ciencias Sociales de la Unesco).

Palabras clave autoras: Subcultura del metal, enseñanza contextualizada.

\section{Contextualized Teaching and Learning using Drama Techniques for Members of the Heavy Metal Subculture}

- Abstract (descriptive): this article describes qualitative research on the inclusion of the heavy metal youth subculture in English as a Foreign Language (EFL) classrooms in Chile, using contextualized teaching and learning as well a drama techniques. Background information related to EFL teaching in Chile is provided followed by a literature review that describes heavy metal as a youth subculture, motivation for learning EFL, contextualized teaching and learning and finally drama techniques. The article goes on to detail the research methodology used (interviews and

Este artículo corto se deriva de un estudio a mayor escala incluido en el Proyecto "Propuesta de Metodologías de Enseñanza Contextualizada del Inglés como Lengua Extranjera para la Inclusión de Estudiantes de tribus urbanas: Metaleros, Skaters, Breakdancers, durante los Ciclos de Bachillerato en la Universidad Austral de Chile." Financiado por la Escuela de Pedagogía en Comunicación en Lengua inglesa de la Universidad Austral de Chile. Código 44151201 realizado desde 14 Marzo 2013-en curso. Investigación cualitativa en el área de Ciencias de la Educación y subárea Educación General.

** Licenciada en Lengua en Literatura Inglesa y Profesora de Inglés de la Pontificia Universidad Católica de Valparaíso (Chile), Doctora en Filología Inglesa por la Universidad de Sevilla (España), Académica Asociada del Instituto de Lingüística y Literatura, Facultad de Filosofía y Humanidades, Universidad Austral de Chile. Orcid: 0000-0001-9117-1152. Índice H5: 2. Correo electrónico: aortizdezarate@uach.cl

*** Licenciada en Educación y Profesora de Comunicación en Lengua inglesa de la Universidad Austral de Chile, candidata a Magíster en Comunicación, por la Universidad Austral de Chile, Docente del Instituto de Lingüística y Literatura, Facultad de Filosofía y Humanidades. Orcid: 0000-0002-9687-4006. Correo electrónico: marianicol.guarda@uach.cl 
non-participant observation) and the results of the study are highlighted. The authors present their conclusions and suggest a pedagogical proposal that brings together all of the concepts examined by this research for the incorporation of heavy metal subculture in EFL lessons.

Key words: subculture, youth, second language instruction, foreign language, English, drama (Unesco Social Sciences Thesaurus).

Authors' key words: heavy metal subculture, contextualized teaching and learning.

\section{Ensino Contextualizado para Membros da Subcultura do Heavy Metal através de Técnicas Teatrais}

- Resumo (descritivo): este resumo descritivo relata a pesquisa qualitativa que visa incluir a subcultura do Heavy Metal em aulas de Inglês como Lingua Estrangeira (ILE) no Chile, usando o ensino contextual e técnicas teatrais. Em primeiro lugar, alguns antecedentes relacionados com à ILE no Chile são concedidos. Em segundo lugar, desenvolve-se uma revisão bibliográfica na qual são descritas as subculturas juvenis, os metaleiros como subcultura, a motivação para aprender ILE, o ensino contextualizado e finalmente técnicas dramáticas. Em terceiro lugar, apresenta-se a metodologia de pesquisa utilizada (entrevistas e observação não participante) e alguns dos resultados obtidos. Finalmente, apresentam-se conclusões e uma proposta pedagógica que utiliza essa pesquisa para incluir a subcultura jovem do heavy metal na aula de Inglês como Língua Estrangeira (ILE).

Palavras-chave: subcultura, juventude, ensino de uma segunda língua, língua estrangeira, inglês, drama (Thesaurus de Ciências Sociais da Unesco).

Palavras-chave autores: subcultura do heavy metal, ensino contextual.

-1. Introducción. -2. Algunos Antecedentes. -3. Subculturas Juveniles. -4. Metaleros. -5. Motivación en los Estudiantes. -6. Enseñanza Contextualizada. -7. Técnicas Teatrales. -8. Metodología. -9. Resultados. $\mathbf{- 1 0}$. Conclusiones. Lista de referencias.

\section{Introducción}

Es un hecho que las y los estudiantes de educación media no logran conseguir los resultados esperados por el Ministerio de Educación chileno en las pruebas estandarizadas. Entre otras razones, se ha demostrado que la desmotivación (Dörnyei, 2008) hacia el aprendizaje de la lengua meta y su cultura es de gran impacto. Esto puede ser debido a que hoy en día las y los jóvenes se sienten más atraídos por sus inclinaciones personales que por su propia educación y la didáctica tradicional no ha podido hacer el puente entre ambos extremos, aparentemente opuestos. John Santrock (2004) destaca que el desempeño de las y los estudiantes se puede optimizar si sus profesores se aproximan a ellos dedicando tiempo para conocer sus predilecciones. Los conceptos "tribus urbanas" o "subculturas" se revelan como una forma de involucrar activamente a las y los estudiantes en su proceso de aprendizaje.
Las subculturas juveniles son un fenómeno de presencia mundial y Chile no es una excepción, ya que existe una gran variedad de las mismas en el país. Andrea Ocampo (2009) se refiere a este tema, mencionando que "Chile se estremece con el rebrote continuo e inminente de las nuevas tribus urbanas" ( $p$. 11). La gran presencia de estas subculturas en el país hace necesaria su integración en el aula, pues esto permite a las y los docentes acercarse a la realidad de su alumnado y conocer sus intereses fuera del aula, logrando así crear clases personalizadas que los haga percibir sus aprendizajes como algo posible de aplicar y comprender aquí y ahora. Dentro de la amplia gama de subculturas juveniles presentes en el territorio nacional, la que se ha seleccionado para este estudio, debido su amplio número de integrantes en el país y a nivel mundial, es la del heavy metal, cuyos miembros se autodenominan "metaleros". Este artículo pone especial énfasis en su manera de vestir, actuar, $\mathrm{y}$ por sobre todo sus inclinaciones, para que 
así profesores de inglés - y de otras materias - puedan usar esta información aplicando educación contextualizada y el uso de técnicas teatrales en adolescentes "metaleros". Así, el principal objetivo de esta investigación es integrar y motivar a estudiantes pertenecientes a la subcultura del heavy metal para que participen en su aprendizaje de marea activa en el aula de inglés como lengua extranjera (ILE) utilizando enseñanza contextualizada y técnicas teatrales en conjunto. Esta propuesta se desprende del hecho de que pertenecer a una subcultura juvenil conlleva características específicas que los integrantes de la misma suelen adoptar como propias. Si además de esto se pudiesen incluir metodologías de enseñanza innovadoras, la motivación del alumnado aumentaría de forma considerable.

La metodología para desarrollar este trabajo es, la revisión bibliográfica en la cual se definen las subculturas juveniles, se determinan las características de los metaleros (incluyendo elementos como vestuario, música, eventos, etc.), se definen diferentes tipos de motivación en el contexto estudiantil y se proponen estrategias para fomentar la motivación intrínseca en estudiantes adolescentes "metaleros". Al mismo tiempo, se exponen en detalle elementos que componen tanto la enseñanza contextualizada como las técnicas teatrales para la enseñanza de ILE. Más tarde, se expone brevemente el estudio de campo que se llevó a cabo en la ciudad chilena de Valdivia, en cuyo contexto se estudió el universo metalero local, sus características, actividades y preocupaciones principales por medio de entrevistas y observaciones no participantes. Finalmente, se apuntan algunas ideas que propendan a desarrollar una propuesta pedagógica para la inclusión efectiva de esta subcultura a la enseñanza del inglés por medio de la enseñanza contextualizada y las técnicas teatrales para la enseñanza del ILE.

\section{Algunos Antecedentes}

La motivación en el estudiantado es un factor fundamental para que puedan aprender un nuevo idioma (Richard-Amato, 2003). En Chile, especialmente en la educación secundaria, existen graves problemas en relación a este tema. Felipe Ferrada y José Sanhueza (2009) mencionan que los adolescentes locales presentan elevado desinterés hacia su aprendizaje en la escuela, y una de las razones más importantes de esta indiferencia es el hastío que producen las metodologías utilizadas por sus profesores, las cuales tienden a ser monótonas y relacionadas únicamente con la teoría ( $\mathrm{p}$. 81). La consecuencia de esta falta de interés se traduce en resultados muy por debajo de lo exigido por el Ministerio de Educación chileno (Mineduc) en las pruebas estandarizadas, tales como el Simce inglés, el cual durante los años 2010 y 2012 demuestra que, una vez que los estudiantes chilenos egresan de la enseñanza media, la gran mayoría de los mismos no es capaz de conseguir siquiera un nivel básico de inglés (Ortiz de Zárate \& Lizasoain, 2013).

En respuesta a los bajos resultados obtenidos, el Mineduc chileno invierte en la implementación de medidas para mejorar la calidad docente. Tales medidas incluyen ejemplos como el Programa Inglés Abre Puertas (Piap), con iniciativas como Redes Docentes de Inglés (RDI), Campamentos de Invierno y Verano en Inglés, Competencias escolares como Spelling Bee, Public Speaking, Debates, entre otras. Sin embargo, los resultados siguen siendo deficientes. Esto se debe nuevamente a la insistencia en metodologías tradicionales, como el método Gramática-Traducción o Grammar-Translation Method, el cual solo desarrolla habilidades receptivas, limitando la producción y por ende la comunicación en inglés por parte de los estudiantes (Ortiz de Zárate \& Lizasoain, 2013). Es por esto que Ferrada y Sanhueza (2009) incentivan el uso de metodologías novedosas que despierten motivación por aprender en los alumnos.

Es importante mencionar que, en el caso chileno, las subculturas juveniles no son una prioridad para el país. De hecho, como menciona Ocampo (2009), Chile "las conoce de reojo, pero las detesta de frentón" (p. 11). Por ejemplo, a la hora de pensar en políticas públicas dirigidas a la juventud, éstas "siguen siendo restrictivas con las aspiraciones juveniles, además de ello no se toman en cuenta sus manifestaciones culturales y contraculturales" (Sánchez, 2007, p. 111). Por ende, se torna relevante dar el primer paso 
y comenzar a entregarle prioridad a aquello que la juventud desea.

Este artículo propone innovar en la sala de ILE, pues la baja motivación en los estudiantes es uno de los principales propulsores de los negativos resultados obtenidos por parte de las y los estudiantes adolescentes chilenos. Los antecedentes analizados evidencian que uno de los principales causantes de la baja motivación en los estudiantes chilenos es la falta de innovación en el aula. Por consiguiente, si las y los docentes de inglés tomaran las atracciones de los estudiantes en cuenta y, en conjunto con eso, dejaran de lado las metodologías tradicionales e innovaran en sus clases, esto permitiría que la motivación aumente. En consecuencia, al proponer en este estudio la integración de las subculturas en el aula, las técnicas y metodologías de enseñanza innovadoras (técnicas teatrales y enseñanza contextualizada) funcionan como un conjunto que muestra a las y los estudiantes que aprender ILE puede hacerse de manera efectiva (mejorando resultados en pruebas estandarizadas, pero sobre todo a nivel comunicacional), dinámica $\mathrm{y}$ atractiva para ellos.

\section{Subculturas Juveniles}

Las subculturas juveniles son un fenómeno en todo el mundo. Desde la segunda guerra mundial han surgido como rebeldes en defensa de la innovación, creando nuevas formas culturales en respuesta a las condiciones de la vida urbana que cambia día tras día (Feixa, 2006). Raúl Olguín (2007) las define como comunidades urbanas que se encuentran en constante desarrollo (p. 9) y se encuentran principalmente en grandes ciudades en las que los jóvenes son un importante actor social. Tania Arce (2008) reúne algunas de las definiciones de tribus urbanas o subculturas juveniles, mencionando que, por ejemplo, se les puede describir como expresiones colectivas en las que los jóvenes construyen sus formas de vida, mostrándose como pequeñas sociedades juveniles. También indica que se les ha definido como identidades que se construyen y expresan durante el tiempo libre o que son grupos de jóvenes adolescentes que usan ropa similar y comparten los mismos hábitos (Arce, 2008). Feixa (2006), por su parte, describe las subculturas juveniles como heterogéneas, pues los estilos de cada una tienden a influenciar a las otras dependiendo de la manera en la que se relacionan, por lo que es común que los jóvenes creen sus características particulares de manera híbrida dependiendo de sus gustos musicales o de las subculturas con las que se reúnan. Esto da como resultado procesos identitarios disímiles que son integrados por variados elementos tomados de la música, la moda, el lenguaje, actividades culturales, etc. (Feixa, 2006, pp. 108-109). Como menciona Olguín (2007), estos grupos juveniles se encuentran en permanente cambio, por lo que nuevas subculturas surgen constantemente; algunas desaparecen tan rápido como llegaron (como por ejemplo el caso chileno de los "Pokemones") mientras que otras pueden perdurar por mucho tiempo (como los Metaleros).

Las subculturas juveniles se encuentran en todo el mundo. Según Carlos Belmonte (2010), esto es producto de los medios masivos de comunicación. Los lugares de origen de las subculturas juveniles más expandidas a nivel mundial suelen ser países tales como los Estados Unidos o Inglaterra. Es gracias a los medios de comunicación masiva que las subculturas creadas son exportadas $y$ asimiladas por jóvenes de diferentes lugares, quienes siguen una estructura general similar a la de origen (Belmonte, 2010, p. 54). Sin embargo, esto no significa que pertenecer a una cierta subcultura en Inglaterra sea igual que pertenecer a la misma subcultura en un país como Chile (Feixa, 2006). Esto se debe a que las subculturas juveniles tienen que ser entendidas como una "manifestación de la capacidad creativa y no solamente imitativa de los jóvenes" (Feixa, 2006, p. 21), puesto que los jóvenes del resto del mundo no necesariamente absorben lo que importan, sino que adaptan las nuevas propuestas, las reutilizan y generan producción cultural propia (Olguín, 2007). La elección de estilo no debe ser confundida con un fenómeno de moda introducido por el mercado ni con la imitación pasiva de los ídolos del rock o de las películas, puesto que los jóvenes construyen sus subculturas tomando elementos 
que van desde el comportamiento a los gustos musicales, transformándolos en prácticas propias (Feixa, 2006), ya que le entregan un significado particular a cada componente (Kristeva, en Weinstein, 2000; Feixa, 2006; Larsson, 2013).

Todas las subculturas juveniles poseen sus propias características y modos de vida. Feixa (2006) enumera y describe algunas, las que incluyen elementos como: códigos de comunicación, apariencia, música, territorio, producción cultural y actividades focales. En el caso de los códigos de comunicación, estos incluyen palabras, frases, e incluso entonaciones que pueden ser inventadas por los jóvenes de la subcultura, por lo que el autor menciona que “es preciso captar su código de comunicación, pues lo que desde fuera puede parecer un caos de ruido... desde dentro se percibe como un sistema altamente organizado y coherente" (Feixa, 2006, p. 138). En el caso de la apariencia, es esta la que más capta la atención de quienes son ajenos a la subcultura, por lo cual es lo que, a simple vista, los diferencia del resto (Belmonte, 2010). Según Feixa (2006), "el estilo puede definirse como la manifestación simbólica de las culturas juveniles, expresada en un conjunto más o menos coherente de elementos materiales e inmateriales, que los jóvenes consideran representativos de su identidad como grupo" (Feixa, 2006, p. 118). Los jóvenes de las diferentes subculturas usan elementos tales como ciertos cortes de pelo, ropa, accesorios, entre otros, para sentirse identificados. Sin embargo, su apariencia no debe ser confundida con uniformes estandarizados, pues los diferentes elementos son utilizados de forma creativa, ya que cada ítem posee un significado (Feixa, 2006). En el caso de la música, esta "es una de esas herramientas que brinda al ser humano la posibilidad de apreciar, contemplar, comprender, crear, recrear, llenar de sentido y significación su propia existencia" (Sánchez \& Acosta, 2008, p. 123). Para muchas subculturas, escuchar y producir cierto tipo de música en particular les habilita para identificarse a sí mismos como individuos y como grupo humano. En el caso del territorio, apropiarse de ciertos espacios urbanos les permite reunirse y delimitar sus fronteras grupales (Cohen en
Feixa, 2006). La producción cultural incluye elementos tales como fanzines, graffiti, tatuajes, etc. Estos suelen ser productos dirigidos a grupos pequeños de personas (solo hacia la subcultura) y son de escasa circulación. Finalmente, las actividades focales son aquellas actividades características de cada subcultura, y usualmente incluyen actividades de esparcimiento, tales como ir a algún bar (Thornton, 1996) o a eventos en particular.

Las subculturas juveniles son un fenómeno complejo, pero su origen y mecánica resulta de gran utilidad a la hora conocer a nuestros estudiantes y sus diferentes tendencias. Este conocimiento es una herramienta de enseñanza muy poderosa para motivar al estudiantado que pertenece a alguna subcultura juvenil. Así, es posible hacer uso de esta información para aplicar la enseñanza contextualizada dirigida a cada subcultura en conjunto con las técnicas dramáticas para innovar en la sala de clases y conseguir motivar al alumnado a aprender. Como ya hemos mencionado, en este artículo en particular, aquella subcultura que atrae nuestro principal interés es la del heavy metal debido a su gran presencia a nivel tanto mundial como local y a sus, muchas veces masivas, manifestaciones culturales.

\section{Metaleros}

Los metaleros han estado presentes en todo el mundo por más de 40 años, y su subcultura va mucho más allá que el simple hecho de seguir un estilo musical, pues se transforma en su forma de vida ya que se sienten uno con la música (Larsson, 2013) y esta característica no es simplemente una "moda de adolescentes". En consecuencia, no es difícil encontrar metaleros desde alrededor de los 13 años hasta los 40 e incluso más. Esta subcultura se basa en el género musical denominado heavy metal, el cual los unifica (Weinstein, 2000) y les entrega una serie de rasgos que los distinguen del resto de los jóvenes.

La subcultura del heavy metal surgió durante los años 70, luego del movimiento contracultural hippie. Inicialmente, los pertenecientes a la subcultura del heavy metal eran jóvenes varones de clase trabajadora, 
quienes comenzaron a rebelarse contra la sociedad al ser privados de privilegios y/o de un futuro prometedor. Es por esto que tomaron y adaptaron características de otras dos subculturas: la hippie y la motoquera. Adoptaron el cabello largo, la vestimenta casual, las drogas y la música psicodélica hippie mientras preservaron el poder físico epitomizado por las imágenes de las bandas motoqueras rebeldes (Weinstein, 2000).

La plataforma del heavy metal (base de la subcultura) es el rock, cuyas raíces son el blues y la música de esclavos. En conjunto con esto, el heavy metal también posee tintes de algunos autores de música clásica como Wagner y Paganini, por ejemplo, pues incluye sonidos duros y oscuros y características de vocalización muy teatrales relacionadas con la opera (Dunn, McFayden \& Feldman, 2005, 00.14.46-00.19.00; Chapman, Dunn \& McFayden, 2011a, 00.11.35-00.18.43; Arnett, 1996, Weinstein, 2000). Actualmente, la música heavy metal es un género musical duro, áspero y agresivo que utiliza temáticas rebeldes que giran en torno a problemáticas consideradas tabú por la sociedad (Saouma, Molpheta, Pille $\&$ de Klepper, 2007, p. 10). El heavy metal se caracteriza por ser rápido, fuerte y emocional, para lo cual se requiere mucha habilidad por parte de la banda. Este tipo de música ha devenido en una enorme cantidad de subgéneros, los cuales poseen diferentes sonidos, tópicos, e incluso diferentes looks (Dunn et al., 2005, 00.35.13-00.36.03). Algunos de los sub-géneros más conocidos incluyen el Black Metal (en el que se incluye el Viking Metal), Death Metal, Doom Metal, Industrial Metal o Metal Industrial, Power Metal, Progressive Metal o Metal Progresivo, Thrash Metal, entre muchos otros (Bukspan, 2012). Existen muchas bandas latinoamericanas tales como Polimetro (Chile, Metal Progresivo), Angra (Brasil, Metal Progresivo/ Power Metal), Rata Blanca (Argentina, Heavy Metal), Sepultura (Brasil, Thrash Metal), pertenecientes a variados subgéneros. Sin embargo, para propósitos de esta investigación se buscan grupos que se enfoquen en la lengua y la cultura inglesa para así ser utilizados dentro de la sala de ILE con el objeto de incentivar aprendizaje de dicha lengua.
Un componente muy importante del heavy metal son las letras y temáticas de las canciones, las cuales varían desde el cristianismo hasta el sexo, dependiendo de la rama del metal (Kotarba \& Wells, en Weinstein, 2000). Algunas de las más comunes y de las cuales se puede sacar más provecho desde el ámbito pedagógico son aquellas que hablan de temas medievales, relacionados con caballeros, mitología nórdica, e incluso con literatura de fantasía que incluye autores tales como J. R. R. Tolkien, Edgar Allan Poe, Shakespeare, Homero, entre otros (Weinstein, 2000; Arnett, 1996; Saouma et al., 2007). Aquellos subgéneros del metal que tratan estas temáticas son el Power Metal, Swedish Metal y el Metal Noruego. Estos se caracterizan por ser muy melódicos, lo cual permite que los fans sigan sus letras al compás de la música (Chapman, Dunn \& McFayden, 2011b, 00.03.40-00.05.00). El Power Metal está mucho más relacionado con la fantasía, mientras que el Swedish Metal y el Metal Noruego están fuertemente enraizados en la historia y mitología de los países nórdicos, tales como los vikingos y sus creencias religiosas, lo cual demuestra el gran orgullo que poseen las bandas por el pasado de sus lugares de origen (Saouma et al., 2007). Es por esto que tanto las bandas como los fans de estos subgéneros se disfrazan y usan armaduras y espadas de época, junto con barbas y ropajes vikingos en ocasiones especiales como tokatas, convenciones y encuentros (Saouma et al., 2007; Chapman et al., 2011b, 00.05.08-00.06.19).

Existen variados elementos que caracterizan a la subcultura metalera. Una de las particularidades que más valoran es el uso del pelo largo, básicamente porque no puede ser ocultado ni disimulado, lo que demuestra un real compromiso con la subcultura (Weinstein, 2000). Lo mismo se aplica a los tatuajes, ya que el hecho de que sean permanentes demuestra la lealtad de los sujetos. Algunos de sus diseños se relacionan con criaturas medievales, como los dragones, y suelen ser ubicados en partes del cuerpo donde puedan apreciarse con facilidad (Weinstein, 2000). Su vestimenta también es muy característica. El elemento que más resalta son las camisetas negras con grandes estampados de los logos de sus bandas 
favoritas, que se lucen con orgullo en el pecho o en la espalda. Además, usan jeans, botas negras o zapatillas, y chaquetas que pueden ser de cuero negro o de jeans, las que generalmente tienen parches o iconos relacionados con la subcultura (Weinstein, 2000; Castillo, 2002; Arnett, 1996; Bukspan, 2012). Aunque en su gran mayoría los seguidores del heavy metal son hombres, las mujeres también pertenecen a esta subcultura, pero su presencia suele ser menos llamativa. Estas suelen tener dos "opciones" de vestimenta: la primera es similar a la masculina usando jeans y camisetas negras de sus bandas favoritas, y la segunda es emular a la bitch goddess que suele aparecer en los videos musicales de las bandas, lo cual implica minifaldas de cuero, medias rotas, zapatos tacón de aguja, y camisetas ajustadas y reveladoras (Bashe, en Weinsten, 2000). Ambas "opciones" son "creadas por el código masculinista: ya sea usando la misma vestimenta que sus pares masculinos o usando la ropa que cumple con la fantasía masculina" (Weinstein, 2000, p. 134).

En resumen, los metaleros son una subcultura que ha estado presente en el mundo desde los setentas, y poseen particularidades determinantes. Su música, el heavy metal, es la base de la subcultura y trata variadas temáticas, entre las cuales destacan los temas medievales y la fantasía, como se mencionó anteriormente. Como muchas subculturas, sus manifestaciones culturales suelen ser reprimidas en contextos más tradicionales. Sin embargo, conocerlos resulta muy útil a la hora de intentar incluirlos en las actividades que se llevan a cabo en la sala de clases, pues integrando sus intereses como subcultura, la enseñanza contextualizada y las técnicas dramáticas se logra llegar no solo al público de los metaleros en particular sino a todos los y las adolescentes en el aula, quienes utilizan la ocasión para aprender de esa subcultura y constatan que el o la docente tiene interés genuino en su integración lo que, sin ninguna duda, los motivará a participar de manera activa en su aprendizaje.

\section{Motivación en los estudiantes}

La motivación en el contexto escolar consiste en el deseo de las y los estudiantes de invertir su tiempo y esfuerzos en clases y/o actividades de aprendizaje (Brophy, 1998), en este caso, de la lengua extranjera inglés. La motivación es de gran importancia ya que es la que se encarga de despertar el deseo de aprender en el alumnado. Dörnyei (2008) confirma este hecho, mencionando que la motivación "posee un rol muy importante a la hora de determinar el éxito o el fracaso del aprendizaje de cualquier lengua" (p. 2). Esto se debe a que si existe una falta de motivación por parte del alumno, incluso a los más dotados les costará prestar atención (Dörnyei, 2008). Si las y los profesores invierten un gran esfuerzo en planificar clases interesantes para los estudiantes, es posible despertar su motivación (Norris-Holt, 2001). En un principio son las y los docentes quienes han de poner el mayor esfuerzo para despertar la motivación del alumnado, pero una vez que se ha logrado este cometido, el estudiantado comienza a interesarse de manera autónoma.

En esta misma línea, la motivación puede ser clasificada como intrínseca y/o extrínseca. La primera es aquella que proviene de los inclinaciones propias de cada estudiante (Lumsden, en Lile, 2002). Esto significa que el alumnado estudia porque desea hacerlo, y no porque se sienta obligado (Brophy, 1998). La motivación extrínseca, por su parte, depende de factores externos al estudiante, como son los incentivos o simplemente tener buenas calificaciones. La motivación extrínseca es más fácil de despertar en el estudiantado que la intrínseca; sin embargo, sus efectos no son duraderos. Por su parte, la motivación intrínseca es más compleja de fomentar, pero sus resultados pueden llegar a marcar a un estudiante de por vida.

Para fomentar la motivación intrínseca de manera exitosa existen cuatro métodos que resultan de utilidad. El primero es evitar la monotonía, lo cual se consigue variando e innovando lo más posible en las clases (Dörnyei, 2008). El segundo es introducir los contenidos de una manera atractiva, para así lograr estimular al alumnado a través de, por ejemplo, actividades desafiantes, graciosas, o competitivas (Vaello, 2011; Dörnyei, 2008). El tercero es involucrar a las y los estudiantes activamente en la clase, lo cual puede lograrse 
a través de actividades en las que deban usar tanto su mente como su cuerpo (Dörnyei, 2008). El cuarto es utilizar materiales que estén relacionados con las realidades de los estudiantes y que sean atractivos para ellos, para que así puedan ver la real importancia de aprender tales contenidos (Dörnyei, 2008, p. 63). Para lograr esto es necesario que el profesor conozca muy bien a sus estudiantes, tanto sus realidades como sus ambiciones.

Como es posible apreciar, la motivación en el alumnado cumple un rol vital en su proceso de aprendizaje. Existen varias formas de fomentar su motivación, y para lograrlo es muy relevante que las preocupaciones de los y las jóvenes estudiantes se integren en el aula con vías a incentivar la motivación intrínseca. Esto puede lograrse conociendo un poco más acerca de las subculturas juveniles, las cuales poseen gran presencia a nivel mundial. Como ya se ha dicho, los metaleros poseen ciertas características que pueden ser fácilmente llevadas al aula.

\section{Enseñanza Contextualizada (CTL)}

La enseñanza contextualizada o CTL (debido a su nombre en inglés Contextualized Teaching and Learning), es una metodología de enseñanza que ha dado resultados positivos no solo en la enseñanza de una segunda lengua, sino que en cualquier área. Esta consiste en crear una relación entre los contenidos que se desean enseñar y situaciones que son relevantes para las y los estudiantes (Baker, Hope \& Karandjeff, 2009b). Su utilidad radica en que los contenidos son más accesibles para el alumnado ya que son explícitamente relacionados a situaciones de la vida real a través de actividades prácticas, lo cual se lleva a cabo con escenarios que son de su interés, ya sean sus preferencias personales (ej.: sus hobbies) o a través de la carrera que estudian (ej.: mecánica) (Mazzeo, en Baker, Hope \& Karandjeff, 2009a).

Esta metodología de enseñanza es utilizada en todo el mundo incluyendo Latinoamérica. Sin embargo, la gran mayoría de las investigaciones realizadas en torno al tema se relacionan con la enseñanza de ciencias. Karina de Freitas y Arnaldo Alves (2010) son un ejemplo de este tipo de estudio en Brasil en su artículo "Reflexiones sobre el papel de la contextualización de la enseñanza de ciencias". En el contexto chileno específicamente, es posible encontrar ejemplos tales como la contextualización para la enseñanza de la física (Rioseco \& Romero, 1999) para la educación ambiental (Figueroa, 2010) o la enseñanza de la química (Reinoso, 2012). Sin embargo, ninguno de ellos puede ser aplicado al área del inglés.

El objetivo principal de la enseñanza contextualizada es generar interés y motivar a las y los estudiantes, lo cual se consigue a través la contextualización de los contenidos. Así, los y las jóvenes logran comprender la importancia de lo que están aprendiendo, ya que pueden observar que los nuevos contenidos que aprenden son efectivamente utilizados en el mundo real. Cuando la información se enseña sin un contexto real, las y los estudiantes suelen tener dificultades para relacionar la teoría con la práctica, ya que no se les entrega ninguna conexión que les ayude a comprender el material entregado (Baker et al., 2009a).

La enseñanza contextualizada se basa en varias teorías. Una de estas es el constructivismo, ya que a través de la práctica el alumnado va construyendo su propio aprendizaje, y va a su vez actualizándolo en la medida que sus experiencias diarias van aumentando (Hein, 1991). Además recoge ideas de la teoría de la motivación, ya que Predmore (2005) afirma que a través del uso de esta metodología los educandos se interesan y se motivan más a aprender, debido a que pueden ver la importancia de los contenidos en el mundo real. Otra de las teorías en las que se asienta la enseñanza contextualizada es la teoría de aprendizaje social, la cual considera que las personas aprenden las unas de las otras (Abbott, en University of South Alabama, s. f., par. 2), lo cual se genera a través de la colaboración en el trabajo grupal. La enseñanza contextualizada también involucra los estilos de aprendizaje, ya que a través de estos los profesores pueden hacer uso de variados métodos pedagógicos que permiten contextualizar las clases, ya sea a través del trabajo en grupo o actividades prácticas manuales (Predmore, 2005).

Esta metodología de enseñanza ha generado muy buenos resultados. El hecho de 
que los contenidos a enseñar sean entregados a través de un contexto relevante para el alumnado les ayuda a crear conexiones entre la teoría y la práctica. Es por esto que la enseñanza contextualizada ayuda a que las y los estudiantes adquieran los conocimientos de una manera más dinámica, pues estos pueden ver la importancia de los contenidos en el mundo real. Consecuentemente, si esta metodología innovadora es utilizada en conjunto con las técnicas teatrales para enseñar integrando las preferencias del alumnado (como las subculturas), lo cual es parte fundamental de la enseñanza contextualizada, la motivación por aprender inglés irá aumentando progresivamente.

\section{Técnicas Teatrales}

Las técnicas teatrales, consideradas como una metodología de enseñanza no tradicional, no son nuevas en el mundo de la pedagogía, lo cual no significa que sean tradicionales. De hecho, existen varios autores y autoras en el mundo que han estudiado y han sacado provecho de ellas en la enseñanza del inglés, obteniendo resultados positivos (Maley \& Duff, 1977, 1982; Maley, 2000; McGregor, Tate \& Robinson, 1977; Kao \& O’Neill, 1998; Heldenbrand, 2003; Athiemoolam, 2004; Schreiber \& Barber, 2005). En Chile, sin embargo, existen muy pocos estudios en el área, existiendo solo algunos ejemplos de estudios de caso tales como los de Ortiz de Zárate (2009a, 2009b, 2012) Lizasoain, Ortiz de Zárate, Walper y Yilorm (2011), y Ortiz de Zárate, Walper, Aros, Hidalgo, Siebert \& Rojas (2014). Cabe destacar que las técnicas teatrales no deben ser confundidas con el teatro. Existen investigaciones que giran en torno al uso del teatro pero no de técnicas teatrales en subsectores que se encuentran estrictamente relacionados con teatro, tales como lenguaje y comunicación o talleres de teatro. Sin embargo, encontrar estudios que giren en torno al uso de técnicas dramáticas es muy difícil, especialmente en Chile y/o en otros subsectores.

Junto con muchas otras metodologías, las técnicas teatrales son consideradas como no tradicionales. El nombre de estas técnicas suele llevar a pensar que el objetivo solo se trata de actuar obras de teatro completas frente a una audiencia, pero no es así, puesto que las técnicas dramáticas van mucho más allá (Maley \& Duff, 1977, 1982; Maley, 2000). Estas incluyen role-plays, juegos, competencias, escenarios, interpretaciones, mímicas, improvisaciones, entre otras actividades (Boal, 1992; Zyoud, 2010) que permiten a docentes de todas las áreas hacer uso de ellas para introducir, enseñar y reforzar contenidos de su subsector. Estas metodologías han demostrado ser muy útiles tanto para aprender una segunda lengua como para desarrollar habilidades para la vida, tales como competencias sociales, de autoconocimiento, y del desarrollo de la identidad.

Las técnicas teatrales poseen varias características que resultan de gran utilidad para la enseñanza del inglés como lengua extranjera. Por ejemplo, utilizan diálogos en la lengua objetivo, lo cual constituye el principal propósito de la adquisición de esta lengua: la comunicación. Además de esto, las técnicas teatrales no utilizan solo palabras o estructuras sueltas y aisladas, sino que entregan los contenidos envueltos en un contexto (Maley \& Duff, 1982; Kao \& O’Neill, 1998). En consecuencia, es posible mencionar que las técnicas teatrales pueden trabajar en conjunto con la enseñanza contextualizada, pues permiten integrar la entrega y la práctica de contenidos dentro de un contexto significativo.

Por otra parte, las técnicas teatrales fomentan el desarrollo de habilidades comunicativas, tales como la velocidad de reacción, la sensibilidad hacia los tonos de voz, etc. (Maley \& Duff, 1982). Junto con esto, también se fomenta el lenguaje no verbal (Berry, 1991, 1992, 2001; Maley, 2000; Neill \& Caswell, 2005; Boston \& Cook, 2009; Swale, 2009; Hamp-Lyons, 2012; Uhler, 2012), ya que combina la comunicación verbal y corporal, lo cual es imprescindible para cualquier interacción social. Además, las técnicas teatrales también ayudan a los estudiantes en la búsqueda de su identidad, parte importante de la adolescencia (Ommanney \& Schanker, 1982; Hamman \& Hendricks, 2005). A través del uso de estas técnicas, los profesores pueden crear un 
ambiente seguro para que los estudiantes puedan experimentar con diferentes roles sociales logrando familiarizarse con ellos (Hamman \& Hendricks, 2005). Esto se puede llevar a cabo a través de role-plays, los cuales además de permitir a los educandos personificar y explorar diferentes roles, les ayuda en su proceso de aprendizaje, pues todo es desarrollado dentro de un contexto y les involucra activamente en la clase. Conjuntamente, quienes son tímidos también pueden verse beneficiados con el uso de estas técnicas, puesto que se potencian habilidades como la expresión, el auto conocimiento, la autoestima, entre otras, que les ayudan a superar la timidez (McGregor et al., 1977). Cabe destacar que las técnicas teatrales también ayudan a desarrollar el trabajo en grupo e incentivan la toma de decisiones, pues deben contribuir y ayudarse los unos a los otros, compartir y aceptar las ideas del grupo, para así poder, por ejemplo, crear un dialogo en conjunto (McGregor et al., 1977).

De esta suerte, el trabajo que permiten desarrollar las técnicas teatrales junto con la enseñanza contextualizada ayudaal estudiantado a ampliar habilidades y competencias necesarias para su educación. Esto se debe a que ambas metodologías entregan contenidos dentro de un contexto relevante y real. Entonces, si ambas metodologías fuesen reunidas en el contexto del interés por la subcultura del heavy metal, estas podrían ayudar a sus adeptos a participar y sentirse más motivados a adquirir la lengua objeto de manera activa.

\section{Metodología}

Esta investigación cualitativa (Nunan, 1992) se encuentra bajo el alero de otra de mayor envergadura en la que se investigan los intereses de variadas subculturas en la localidad de Valdivia, Región de Los Ríos, Chile, dentro de las cuales se encuentran Skaters, Breakdancers y Metaleros. En este artículo hemos decidido presentar la cultura metalera, puesto que se encuentra presente a nivel mundial y ha demostrado ser estable en el tiempo y cantidad de participantes, por lo cual, esta investigación puede ser utilizada tanto en diferentes lugares del mundo como en el presente y el futuro.
Asimismo, los miembros de esta subcultura resaltan en la multitud debido a su vestuario, por lo que pueden reconocerse con facilidad.

A pesar de que existen metaleros de todas las edades, los sujetos estudiados fueron adolescentes desde los 17 a los 25 años de edad. Esto se debe principalmente a que, en el caso de Chile, los adeptos a las subculturas juveniles que estudian en la enseñanza secundaria suelen verse impedidos de expresarse abiertamente en lo que a vestuario se refiere ya que se exige que el estudiantado utilice un uniforme institucional. Se utilizaron sujetos pertenecientes a la enseñanza superior, pues estos poseen mayor libertad de expresión en lo que a su imagen respecta. Los sujetos fueron estudiados en entornos públicos universitarios y en los lugares de la ciudad en los que se suelen reunir. A pesar de que esta subcultura se encuentra dominada principalmente por el género masculino, los sujetos de esta investigación incluyen hombres y mujeres. De esta forma, esta investigación de campo dará cuenta de las principales características de los sujetos, las cuales incluyen vestuario, actividades, preferencias musicales, y creencias, entre otros.

Este estudio utilizó dos herramientas de recolección de datos con el objetivo de descubrir qué incentiva a estos jóvenes en la ciudad chilena de Valdivia: la observación no participante y las entrevistas semiestructuradas. En consecuencia, se realizaron observaciones no participantes a miembros de la subcultura heavy metal ya que estas permiten que el estudio se lleve a cabo de una manera poco intrusiva y lo más natural posible (Hancock \& Algozzine, 2006). Es por esto que se observó a 9 sujetos durante un tiempo total de 3.1 horas/ 190 minutos, los cuales fueron distribuidos en varias observaciones que rondan entre los 5 minutos y 1.5 horas dependiendo de cuán accesibles fuesen los sujetos en cada sesión. Al mismo tiempo, se llevó a cabo una serie de entrevistas semiestructuradas con miembros de la subcultura metalera: participantes de la cultura, dueños de tiendas especializadas (quienes se encuentran en permanente contacto con los sujetos) y, finalmente, con un experto en subculturas juveniles (Dr. Yanko González). El principal foco de interés son las motivaciones 
de los sujetos para volverse metaleros, los elementos que han adoptado de la subcultura, las temáticas que les atraen de la música metal, y la manera en la que se sentirían si las ideas propuestas por esta investigación fuesen llevadas a cabo en la sala de clases de ILE.

\section{Resultados}

Los resultados obtenidos en base a las ya mencionadas herramientas de investigación consideran, primero, las actividades focales de la subcultura (tokatas, conciertos, pertenencia a bandas de heavy metal, etc.). Segundo, el consumo de elementos de la subcultura, los cuales incluyen tanto los diferentes subgéneros de la música heavy metal como la ropa y los accesorios. Tercero, se tomó en cuenta el sentido de pertenencia (una de las principales características de todas las subculturas) así como las motivaciones generales de los metaleros, su estilo de vida y las razones que les hacen sentir parte de la subcultura.

En primer lugar están las actividades focales o ritualizadas. Estas son actividades compartidas por los miembros de la subcultura o, en palabras de Alfonso Torres (2006), "se refieren a eventos de carácter cotidiano que tienen un espacio institucionalizado y que se repiten en un formato estandarizado y con cierto rigor" (p. 130). Entre estas fue posible identificar tokatas, conciertos, creación de bandas propias y literatura. Todos los metaleros observados y entrevistados poseían algún tipo de relación con alguna de las actividades focales. Las primeras tres actividades se relacionan con la música heavy metal en sí, base principal de esta subcultura. En el caso de la literatura, lo que más atraía a los sujetos era la fantasía épica (como los libros de J. R. R. Tolkien), la historia nórdica (vikingos) o el ocultismo.

En el caso del consumo de elementos de la subcultura el más recurrente fue la estética metalera con base en la música heavy metal (videos, bandas y metaleros pertenecientes a su círculo). Es necesario destacar que la música heavy metal posee múltiples sub-géneros, pero aquellos con más adeptos en la localidad de Valdivia son los sub-géneros como el Heavy, Death, Black, Power, Folk, Goth y Thrash
Metal, los cuales abordan temáticas desde la religión a la fantasía épica. En particular, el subgénero más mencionado por los entrevistados fue el Power Metal, ya sea por sus temáticas relacionadas con la fantasía épica o por su ritmo melódico.

Como en toda subcultura, un sello de los metaleros es manifestar de una manera $u$ otra su estética regional, nacional e internacional. Dicha estética incluye ropa negra, cabello largo, tatuajes y camisetas de sus bandas favoritas. Dependiendo de factores como la edad, el nivel sociocultural y las restricciones de su entorno, los sujetos incluían alguno de estos elementos adaptándolos e integrándolos a su construcción de identidad, demostrando la capacidad creativa de la subcultura.

Finalmente, el sentido de pertenencia, característica fundamental en las subculturas juveniles, se manifiesta en los metaleros como un estilo de vida que perdura incluso hasta la adultez. La música es la columna vertebral de esta subcultura y ayuda a los sujetos a construir un sentido de identidad, especialmente durante la adolescencia. Es a través de la música que los metaleros se relacionan con el mundo, y debido a la amplia gama de sub-géneros musicales, son capaces de construir diferentes realidades regionales.

Cuando se le planteó a los sujetos entrevistados la idea general propuesta por esta investigación y se les consultó su opinión sobre la posible aplicación de la propuesta, los entrevistados demostraron un abierto interés por participar. Mencionaron, por ejemplo, que consideraban que su interés por adquirir la lengua objeto aumentaría de manera considerable si se aplicaran las técnicas teatrales en conjunto con la enseñanza contextualizada en torno a temas atractivos para su subcultura.

\section{Conclusiones en vías a una Propuesta Pedagógica}

Como se ha planteado en este artículo, los estudiantes adolescentes chilenos presentan una gran falta de motivación. Se han sindicado a las metodologías de enseñanza tradicionales como las principales responsables de este fracaso. Dicha situación hace necesario que las y los 
docentes innoven en su quehacer cotidiano. Este artículo propone que para esa innovación se utilice la enseñanza contextualizada en conjunto con las técnicas dramáticas. Junto con esto, se ha observado que se puede fomentar la motivación intrínseca de los estudiantes gracias a la inclusión de sus preferencias en el aula. Estos intereses pueden ser identificados e incluidos gracias al estudio de las subculturas juveniles, como es el caso de los metaleros. $\mathrm{Si}$ algunas características particulares de la subcultura son utilizadas como parte del contexto significativo que requiere la enseñanza contextualizada y las técnicas dramáticas, la motivación en los estudiantes de la subcultura se verá influenciada de manera positiva. Esto se debe a que los y las adolescentes podrán reconocer y comprobar aplicaciones reales para el idioma en un contexto relevante para ellos, como lo es la música heavy metal y sus diferentes temáticas.

En base a los resultados obtenidos por esta investigación, se puede mencionar que incluir a los metaleros en el aula ILE es posible. Para llevar a cabo dicha tarea aquí se entrega una serie de ideas educativas que utilizan las metodologías mencionadas en conjunto, utilizando como base los intereses de la subcultura objeto.

Una manera de incluir a los metaleros a través de la educación contextualizada en conjunto con las técnicas dramáticas es la música. Como se mencionó anteriormente, una de las bases de este método es la entrega de un contexto significativo del uso de los contenidos a enseñar. En este caso, la música es un elemento que demuestra que las palabras a enseñar se utilizan en la realidad por artistas en la música del interés del alumnado metalero. Específicamente, la integración de la música metal en el aula ayuda a lograr la enseñanza contextualizada de manera directa, ya que se utiliza un contexto real del uso del idioma a través de uno de los elementos base de la subcultura del metal. Los seguidores del metal tienen un gran interés por este tipo de música y por ende un contacto directo diario con la misma, por lo cual pueden asociar una inmediata relación de relevancia de los contenidos. Los sub-géneros de heavy metal más recomendables para utilizar en este tipo de actividades son: Power Metal y algunas ramas del Black Metal, como son el Swedish Metal, y Metal Noruego, debido a sus melodías y temáticas. Sin embargo, para poder lograr la inclusión coherente y eficiente de estos contenidos es necesario buscar las canciones adecuadas. Muchas veces, las letras pueden ser incomprensibles debido a la vocalización de los cantantes o porque el volumen de los instrumentos supera el volumen de la voz. También pueden incluirse canciones que no son parte del heavy metal o de sus subgéneros, sino que posean temáticas asociadas a la subcultura (por ejemplo, que hablen de temáticas medievales, mitologías vikingas, batallas épicas, entre otras), como en el caso del Folk Metal.

La integración de técnicas dramáticas tales como los diálogos creados por los mismos alumnos son otro buen elemento que ayuda a complementar la enseñanza contextualizada. Luego, para comprender la letra de las canciones, una manera de fomentar el pensamiento crítico en los alumnos, se puede discutir su temática y que ellos entreguen argumentos basados en la letra para discutir contenidos relacionados a sus realidades socioculturales. Esto puede ser llevado a cabo ya sea como una actividad de grupo-curso, en la que las ideas sean mencionadas y argumentadas de forma colectiva, o como una actividad en grupos más pequeños que lleguen a una conclusión que se comparta frente al resto de la clase. Finalmente, la creación de diálogos basados en la temática de la canción es una actividad que involucra tanto la enseñanza contextualizada como las técnicas teatrales. Ambas estrategias otorgan el uso contextualizado del idioma en base a un tema musical con la creación y representación de un diálogo original, demostrando que el vocabulario en ILE se identifica, adquiere y utiliza dentro de un todo, y no sólo como palabras aisladas.

En caso de que los alumnos no posean un nivel de inglés suficiente para crear diálogos, existen otras técnicas teatrales que se pueden utilizar. La mímica es un elemento divertido, rápido y simple que permite reforzar el vocabulario en las canciones. En esta actividad y otras semejantes se recomienda la participación de equipos que compitan entre sí, 
lo cual puede ayudar a fomentar la motivación del estudiantado, sobre todo en el caso de los adolescentes, quienes suelen ser altamente competitivos. Un tipo de actividad que puede utilizarse como proyecto de final de año es un festival de música, en el cual los alumnos se disfracen como los artistas e interpreten las canciones, ya sea cantando con su voz propia o imitando sobre la pista musical (lip-sync battle) enfatizando palabras y estructuras y utilizando emociones para así poder proyectar la temática de fondo (por ejemplo, demostrando rabia si la temática de la canción es esta). Esto les faculta para incluir elementos tanto lingüísticos como culturales de la lengua objetivo.

Es posible argumentar que los resultados esperables de la aplicación de este planteamiento han de ser positivos, puesto que los mismos sujetos del estudio admitieron un abierto interés por el desarrollo de esta propuesta y mencionaron que su motivación en torno a la adquisición de la lengua objeto aumentaría si se incluyeran rasgos de su subcultura en el aula de ILE. Asimismo, como la subcultura juvenil del heavy metal no es la única, lo que se propone en este artículo puede ser aplicado con otras subculturas, tales como los Punks, Góticos, Otakus, Gamers, etc. Cada subcultura juvenil posee diferentes rasgos que forman parte de su identidad grupal, por lo que es necesario investigar cada subcultura antes de aplicar esta propuesta con diferentes audiencias, incluso aquellas que desaparecen tan rápido como surgen. También se propone fomentar el uso de metodologías de enseñanza innovadoras para la inclusión de las diferentes subculturas y sus intereses en el aula, lo cual permitiría a las y los docentes poseer una gama de estrategias mucho más amplia para adaptarlas a sus propias aulas. Entre más innovación exista, más estudiantes tendrán la oportunidad de romper la monotonía y sentirse motivados a aprender de manera activa no solo una nueva lengua, sino que también otros subsectores.

\section{Lista de referencias}

Arce, T. (2008). Subcultura, contracultura, tribus urbanas y culturas juveniles: ¿homogenización o diferenciación? Revista
Argentina de Sociología, 2, pp. 257-271.

Arnett, J. (1996). Metal heads: Heavy metal music and adolescent alienation. Colorado: Westview Press.

Athiemoolam, L. (2004). "Drama in Education and its Effectiveness in English Second/Foreign Language Classes". First International Language Learning Conference (ILLC), Penang, Malaysia. Recuperado de:

http://www.zsn.uni-oldenburg.de/ download/Logan Malaysia Conference. pdf

Baker, E.; Hope, L. \& Karandjeff, K. (2009a). Contextualized Teaching \& Learning: A Faculty Primer. Recuperado de: http:// www.rpgroup.org/content/ctl-writtenresources

Baker, E.; Hope, L. \& Karandjeff, K. (2009b). Contextualized teaching \& learning: a promising approach for basic skills instruction. Recuperado de: http://www. rpgroup.org/content/ctl-written-resources

Belmonte, C. (2010). Las tribus urbanas: campo virgen en la historia y fértil para la interdisciplinariedad. Cuicuilco, 48, $\mathrm{pp}$. 49-67.

Berry, C. (1991). Voice and the Actor. New York: Wiley Publishing, Inc.

Berry, C. (1992). The Actor and the Text. New York: Applause.

Berry, C. (2001). Text in Action. London: Virgin Publishing.

Boal, A. (1992). Games for Actors and NonActors. London: Routledge.

Boston, J. \& Cook, R. (2009). Breath in Action. The Art of Breath in Vocal and Holistic Practice. London: Kingsley Publishers.

Brophy, J. (1998). Motivating students to learn. Boston: McGraw-Hill.

Bukspan, D. (2012). The encyclopedia of heavy metal. Toronto: Sterling.

Castillo, C. (2002). De las bandas a las tribus urbanas. De la transgresión a la nueva identidad social. Desacatos, 9, pp. 57-71.

Chapman, R.; Dunn, S. \& McFayden, S. (writers); Dunn, S. \& McFayden, S. (directors) (2011a). Pre Metal [Television series episode]. In Banger Films (producer) Metal Evolution. Toronto: Tricon Films \& 
Television.

Chapman, R.; Dunn, S. \& McFayden, S. (writers), Dunn, S. \& McFayden, S. (directors) (2011b). Power Metal [Television series episode]. In Banger Films (producer) Metal Evolution. Toronto: Tricon Films \& Television.

De Freitas, K. \& Alves, A. (2010). Reflexiones sobre el papel de la contextualización de la enseñanza de ciencias. Enseñanza de las Ciencias, 28 (2), pp. 275-284.

Dörnyei, Z. (2008). Motivational strategies in the language classroom. Cambridge: Cambridge University Press.

Dunn, S.; McFayden, S. \& Feldman, S. (producers); Wise, J.; Dunn, S. \& McFayden, S. (directors) (2005). Metal: a headbanger's journey [Motion picture]. Toronto: Seville Pictures, Warner Home Video.

Feixa, C. (2006). De jóvenes, bandas y tribus. Barcelona: Ariel.

Ferrada, F. \& Sanhueza, J. (2009). Adolescencia y motivación educacional: un estudio a Adolescentes de Segundo Año Medio del Liceo Industrial y del Instituto Italia de Valdivia. [Unpublished undergraduate thesis]. Universidad Austral de Chile, Valdivia, Chile.

Figueroa, A. (2010). Las redes complejas de un sistema educativo contextualizado en su entorno. En R. Arrué (ed.) Balance y Perspectivas de la Educación Ambiental en Chile e Iberoamérica, Chile, Tomo I., (pp. 147-157). Santiago de Chile: Gobierno de Chile, Comisión Nacional del Medio Ambiente (Conama).

Hamman, D. \& Hendricks, C. (2005). The role of the generations in identity formation: Erikson speaks to teachers of adolescents. The Clearing House, 79 (2), pp. 72-75.

Hamp-Lyons, E. (2012). "Your Most Essential Audiovisual Aid-Yourself!". English Teaching Forum, 50 (4), pp. 33-35.

Hein, G. (1991). Constructivist leaning theory. Recuperado de: http://www.exploratorium. edu/ifi/resources/constructivistlearning. html

Heldenbrand, B. (2003). Drama techniques in English language learning. Korea TESOL
Journal 6 (1), pp. 27-38. Recuperado de: http://www.kotesol.org/files/u1/ktj6_all. pdf

Kao, S. \& O’Neill, C. (1998). Words into Worlds: Learning a Second Language through Process Drama. Stanford: Alex Publishing Corporation.

Larsson, S. (2013). 'I bang my head, therefore I am': Constructing individual and social authenticity in the heavy metal subculture. Young, 21 (1), pp. 95-110. Doi: 10.1177/1103308812467673.

Lile, W. (2002). Motivation in the ESL Classroom. The Internet TESL Journal, VIII (1). Retrieved from: http://iteslj.org/ Techniques/Lile-Motivation.html

Lizasoain, A.; Ortiz de Zárate, A.; Walper, K. \& Yilorm, Y. (2011). Estudio descriptivo y exploratorio de un taller de introducción a las técnicas teatrales para la enseñanza/ aprendizaje de una lengua extranjera. Estudios Pedagógicos 37 (2), pp. 123-133.

Maley, A. (2000). The Language Teacher's Voice. Oxford: Macmillan-Heinemann.

Maley, A. \& Duff, A. (1977). The use of dramatic techniques in foreign language learning. In English Teaching Information Centre (Etic), British Council (ed.) Games, simulations and role-playing, (pp. 17-33). London: British Council.

Maley, A. \& Duff, A. (1982). Drama Techniques in language learning: a resource book of communication activities for language teachers. Cambridge: Cambridge University Press.

McGregor, L.; Tate, M. \& Robinson, K. (1977). Learning through drama. London: Heinemann Educational Books.

Neill, S. \& Caswell, Ch. (2005). Body Language for Competent Teachers. London: Routledge.

Norris-Holt, J. (2001). Motivation as a Contributing Factor in Second Language Acquisition. The Internet TESL Journal, 7 (6). Recuperado de: http://iteslj.org/ Articles/Norris-Motivation.html

Nunnan, D. (1992). Research methods in language learning. New York: Cambridge 
University Press.

Ocampo, A. (2009). Ciertos ruidos: nuevas tribus urbanas chilenas. Santiago de Chile: Planeta.

Olguín, R. (2007). Ciudad y tribus urbanas: el caso de Santiago de Chile (1980-2006). Diseño Urbano y Paisaje, 4 (10), pp. 3-22.

Ommanney, K. \& Schanker, H. (1982). The stage and the school. New York: McGraw Hill.

Ortiz de Zárate, A. (2009a). Propuesta y Validación del Método de Traducción Teatral 'Escritura en Voz alta', a través de Obras de Teatro británico contemporáneo. Fondecyt $\mathrm{N}^{\mathrm{o}}$ 11090198. Investigadora Responsible Proyecto Fondecyt de Iniciación. No publicado.

Ortiz de Zárate, A. (2009b). Inglés: actuando el lenguaje. Enfoques metodológicos para crear un clima favorable que propicie la enseñanza y adquisición efectiva del inglés como lengua extranjera en comunidades del sur de Chile. DID S-2009-16. Investigadora responsible proyecto Dirección Investigación y Desarrollo UACh. No publicado.

Ortiz de Zárate, A. (2012). Propuesta y validación de metodologías activas para la inclusión de estudiantes con discapacidad visual durante los ciclos de bachillerato en la Universidad Austral de Chile. DEP201201. Directora Proyecto Innovación Docente Dirección Estudios de Pregrado UACh. No publicado.

Ortíz de Zárate, A. \& Lizasoaín, A. (2013). Kelluplay: técnicas dramáticas e instrucción basada en contenidos apoyadas en servicios de la web 2.0 para la adquisición del inglés en estudiantes vulnerables del sur de Chile. Proyecto Fondef. No publicado.

Ortiz de Zárate, A.; Walper, K.; Aros, P.; Hidalgo, D.; Siebert, F. \& Rojas, J. (2014) "El Rol de la interacción significativa y las técnicas dramáticas en la educación de estudiantes con discapacidad visual en la UACh". Colombian Applied Linguistics Journal, 16 (2), pp. 261-276.

Predmore, S. (2005). Putting it into context. Recuperado de:
http://www.wflboces.org/uploads/SDM/ PuttingIt $\% 20$ IntoContext $\% 20$ Full $\% 20$ Article1_05.pdf

Reinoso, J. (2012). Caracterización del proceso de contextualización curricular para la enseñanza de la química: un estudio de caso (Unpublished master's thesis). Universidad Católica de Chile, Santiago de Chile, Chile.

Richard-Amato, P. (2003). Making it happen. New York: Pearson Education.

Rioseco, M. \& Romero, R. (1999). La dimensión afectiva, como base para la contextualización de la enseñanza de la física. Estudios Pedagógicos, 25, pp. 5170.

Sánchez, M. (2007). Thrash Metal: del sonido al contenido: origen y gestación de una contracultura [Unpublished undergraduate thesis]. Universidad de Chile, Santiago de Chile, Chile.

Sánchez, T. \& Acosta, A. (2008). Música popular campesina. Usos sociales, incursión en escenarios escolares y apropiación por los niños y niñas: la propuesta musical de Velosa y Los Carrangueros. Revista Latinoamericana de Ciencias Sociales, Niñez y Juventud, 6 (1), pp. 111-146.

Santrock, J. (2004). Children. New York: McGraw-Hill.

Saouma, R.; Molpheta, S.; Pille, S. \& de Klepper, S. (2007). Cultural heritage and history in the European metal scene. Belvedere Educational Network, Projectbureau.

Schreiber, T. \& Barber, M. (2005). Acting: Advanced Techniques for the Actor, Director, and Teacher. New York: Allworth Press.

Swale, J. (2009). Drama Games for Classrooms and Workshops. London: Nick Hern Books.

Thornton, S. (1996). Club cultures: music, media, and subcultural capital. Middletown: Wesleyan University Press.

Torres, A. (2006). Organizaciones populares, construcción de identidad y acción política. Revista Latinoamericana de Ciencias Sociales, Niñez y Juventud. 4 (2), pp. $167-$ 199.

Uhler, J. (2012). "Essentials for the Teacher's Toolbox". English Teaching Forum, 50 (4), 
pp. 21-32. Recuperado de:

http:/www.southalabama.edu/oll/mobile/ theory_workbook/social_learning_theory. htm

Vaello, J. (2011). Cómo dar clase a los que no quieren. Barcelona: Graó.

Weinstein, D. (2000). Heavy metal: the music and its culture. New York: Da Capo Press.

Zyoud, M. (2010). Using Drama Activities and Techniques to Foster Teaching English as a Foreign Language: a Theoretical Perspective. Retrieved from:

http://www.qou.edu/english/conferences/ firstNationalConference/pdfFiles/ muntherZyoud.pdf 